\title{
Two-Process Model of Sleep
}

\author{
Garima Shukla, Deepti Vibha
}

Department of Neurology, All India Institute of Medical Sciences, New Delhi, India

DOI No: 10.5958/0974-0155.2014.01108.5

Indian J Sleep Med 2014; 9.3, 91-95

\begin{abstract}
The intricate interplay between circadian and ultradian processes and rhythms that determine sleepiness and wakefulness in normal human adults has been widely studied and has been excellently presented in the form of mathematical models interweaving homeostatic, circadian, and ultradian processes. The evolution of knowledge about these and the clinical applications of this view about sleep regulationhave been discussed in this review.
\end{abstract}

\section{Introduction}

$\mathrm{N}$ ormal human sleep, an essential physiologic process, comprises two phases or cycles, rapid eye movement (REM) sleep and non-REM sleep. Sleep is regulated by intricately interrelated activity and complex interplay at different anatomically identifiable centers, and the simultaneous occurrence of various behavioral and physiologic processes.Sleep-wake cycles are determined by synchrony between sleep homeostasis and circadian rhythms. Many models have been developed to explain the delicate interplay resulting in sleepiness or wakefulness, the most accepted among which remains the schema originally proposed by Borbély. ${ }^{1}$

\section{Originally Proposed Model}

One of the major advances in the understanding of sleep physiology was the development of the two-process model of sleep regulation. ${ }^{1,2}$ It was originally described in a rat

\section{Address for Correspondence}

Dr. Garima Shukla

Professor, Department of Neurology

Room No. 2, 6th Floor, Neurosciences Center

All India Institute of Medical Sciences

New Delhi, India

Tel: +911126593785

Email: garimashukla@hotmail.com model and was qualitative in nature. The model consists of a homeostatic process termed process $S$ and an intrinsic circadian pacemaker (process) termed process C. Process $S$ represents a putative drive for sleep that progressively increases in intensity during wakefulness and shows a reduction during (non-REM) sleep. Process $\mathrm{C}$ represents a (nearly) 24-h spontaneous oscillatory variation in the propensity for sleep. These two processes can predict the timing and duration of sleep and the intensity of non-REM sleep. ${ }^{3}$ The two-process model of sleep regulation has been applied successfully to describe, predict, and understand the sleep-wake regulation in a variety of experimental protocols such as sleep deprivation and forced desynchrony ${ }^{4}$.

Much of the research on sleep homeostasis has been possible due to the recognition of the physiologic correlate of sleep propensity, slow-wave activity (SWA) on electroencephalogram (EEG). This has permitted measurement of sleep pressure under experimental conditions in both humans and animals. ${ }^{5}$ Further, an inverse relationship between EEG SWA and brief awakenings during sleep has been observed and established ${ }^{6}$.

Beyond the Borbély model, the mathematical mechanisms and models that account for the complex interplay between circadian, ultradian (physiologic cycles of less than $24 \mathrm{~h}$ duration), and homeostatic aspects of sleep regulation have also been proposed ${ }^{7}$. Nevertheless, the Borbély two-process model is still the most accepted 
theory, which describes the orchestrated balance necessary to achieve a normal sleep-wake cycle. ${ }^{8}$ The original Borbély two-process model was qualitative whereas a quantitative version was established later, based on this. In the latter, process $S$ varied between an upper and a lower threshold that in turn was modulated by a fixed circadian process $\mathrm{C}$. This model has, thus, been able to explain phenomena such as recovery from sleep deprivation, circadian phase dependence of sleep duration, sleep in shift workers, sleep fragmentation during continuous bed rest, and internal desynchronization in absence of cues ${ }^{1}$. The processes thatunderlie sleep regulation are broadly categorized as follows:
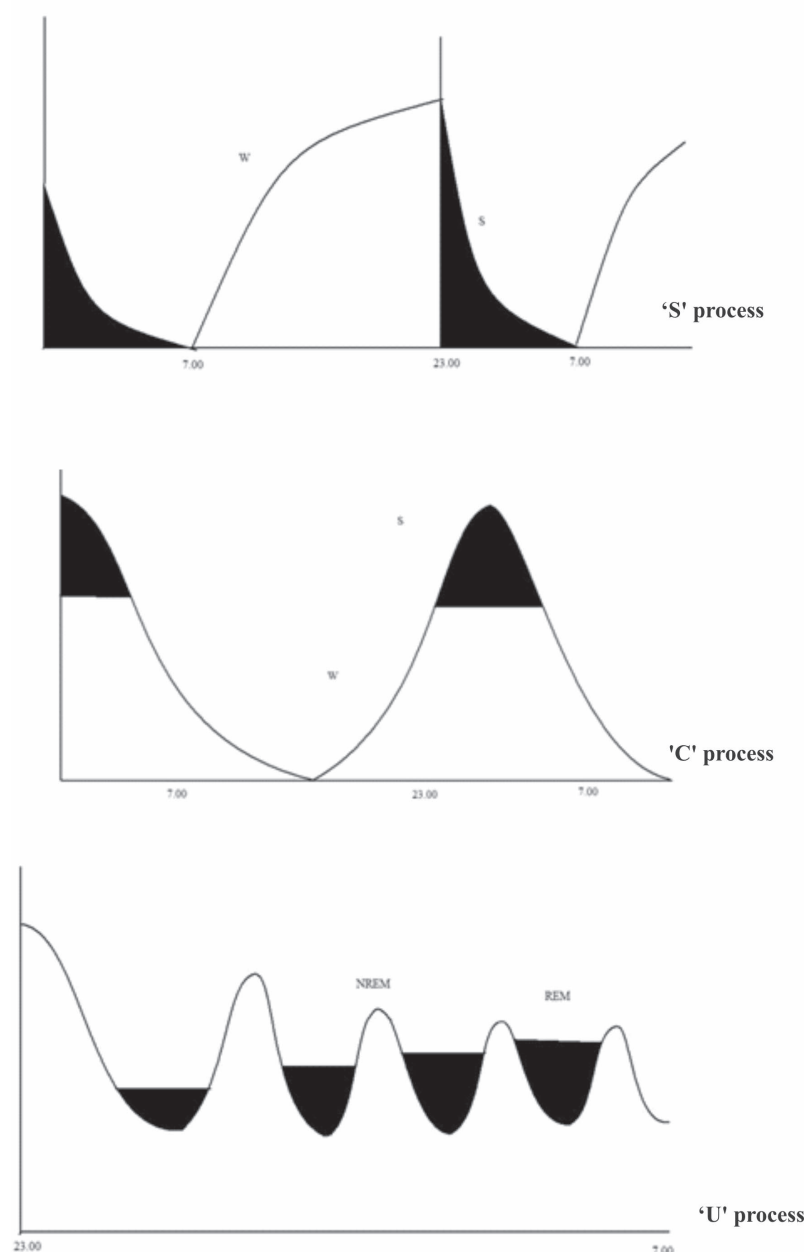

Figure 1: Diagrammatic representation of the basic processes of sleep regulation (modified from Achermann and Borbély ${ }^{7}$ )
1. Homeostatic process $S$ : This process mediates the rise in "sleep pressure" during waking and the dissipation of "sleep pressure" during sleep.

2. Circadian process C: This process operates independently of the duration of wakefulness, cycling in a fixed and rhythmic pattern to promote alertness. Most circadian models assume that multiple oscillators underlie the differences in period and entrainment properties of the sleep-wake cycle and other rhythms (e.g., body temperature).

3. Ultradian process: This process simulates the cyclic alternation of non-REM sleep and REM sleep by assuming a reciprocal interaction of two cell groups. These occur within sleep and represented by the alternation of the two basic sleep states: non-REM and REM sleep. Described as part of a model of ultradian variation of SWA, herein, the change of $S$, not the level of $S$, corresponds to SWA. An REM sleep oscillator triggers the decline of SWA during REM sleep ${ }^{7}$.

\section{Interplay between S and C Processes}

The homeostatic sleep drive is directly determined by the duration of wakefulness. In the morning, with an adequate amount of sleep, process $S$ is at its nadir. As the day proceeds and the duration of wakefulness grows in length, process $S$ or the drive/pressure to sleep increases linearly until the person goes to sleep effectively working to reduce the $\mathrm{S}$ drive. The circadian process $\mathrm{C}$ operates independently of the duration of wakefulness; this process cycles in a fixed and rhythmic pattern to promote alertness (Figure 1). During the day, when the homeostatic sleep drive is mounting, wakefulness is maintained because the circadian process works to offset this rising drive toward sleep. The timing of circadian rhythm and the homeostatic sleep drive normally align to achieve a fixed and consolidated sleep-wake cycle. However, individuals can experience a dip in their circadian alerting drive in the late afternoon, which explains the common after-lunch dip or siesta in alertness ${ }^{9}$. Under conditions of sleep deprivation, the interplay between the homeostatic and the circadian process becomes less coordinated and the sleep-wake state becomes unstable ${ }^{10}$; sleep-deprived individuals showevidence of involuntary sleep intrusions ${ }^{11}$. The circadian after-lunch dip in alertness is amplified in the face of sleep loss ${ }^{12,13}$. 


\section{Evolution of the theory (addition of two processes)}

The two-process model triggered numerous experimental studies, both qualitative and quantitative (using SWA measures), using animal and human subjects. Beyond the two basic models and the third interlaced with them, new extensions have been added such as the W(sleep inertia) ${ }^{14}$ and $Z$ process (which explains regional specificity, with evidence that SWA dissipates more rapidly in frontal derivations on the EEG) ${ }^{15}$.

From analysis of SWA across multiple cortical locations, the dissipation of SWA according to the $Z$ process is expressed more clearly in the frontal regions. It basically encompasses the regional specificity in regulation over different brain areas, in contrast to the global S regulation ${ }^{16,17}$.

Although dealing with individual mathematical models of sleep is beyond the scope of this article, different models have been developed to better explain the C, S, and ultradian processes. The circadian models have been found to be variously dependent on light, split or non-split sleep pattern, sleep timing, temperature rhythm, melatonin, and pineal regulation ${ }^{16-18}$. Studies published as early as 1970 established the suprachiasmatic nucleus of the hypothalamus as the central circadian pacemaker in mammals ${ }^{19}$. This pacemaker comprises individual cells that, when isolated, can oscillate independently with a near-24-h period. The suprachiasmatic nucleus receives direct input from the retina, ${ }^{20}$ providing a mechanism by which entrainment to light-dark cycles occurs. Taken together the studies so far suggest that the overall 24 -h pattern of light and darkness to which humans are exposed plays a critical role in subsequent sensitivity to light exposure, and thus in entrainment ${ }^{21}$. The circadian system of individuals who get little bright-light exposure may become more sensitive to moderate levels of light. Given that most studies show that modern humans get relatively little bright-light exposure and instead spend most of their waking day in light of indoor intensity, these findings may have very important practical relevance for most humans.

Process $S$ is determined by the buildup rate and the saturation level of SWA within non-REM sleep episodes ${ }^{22}$ and also the sleep inertia (process W) ${ }^{7,22}$. Not only the timing of sleep but also the time course of daytime vigilance can be accounted for by the interaction of homeostatic and circadian processes. ${ }^{7}$ The posterior hypothalamus, tuberomammillary nucleus, and regions of the brain stem are all collectively involved in maintaining wakefulness with the release of excitatory neurotransmitters orexin, histamine, and acetylcholine, respectively, to specific cortical and subcortical sites. During wakefulness, adenosine, a nucleoside involved in intracellular energy transfer and storage, continues to build in the system and serves as a soporific neurotransmitter to help transition into the sleep state. Adenosine represents the neurophysiologic marker of the homeostatic sleep drive. Therefore, similar to the process $S$, which increases with the duration of wakefulness, so does the amount of adenosine along the neuroaxis.

The reciprocal interaction models attempt to explain the cyclic alternation of non-REM sleep and REM sleep. While the regulation of non-REM sleep has been extensively modeled by comparing empirical and simulated data in various experimental schedules, relevant and conceptually useful models have been lacking for REM sleep. If REM sleep is homeostatically regulated, it implies that REM sleep propensity accumulates in the absence of REM sleep ${ }^{21}$. The reciprocal interaction model postulates that the non-REM/REM sleep cycle is generated by the reciprocal interaction of two neuronal systems in the brain stem.

Attempts were also made to integrate various concepts into a combined model. While initial simulations showed the feasibility of incorporating homeostatic, circadian, and ultradian factors regulating nighttime and daytime sleep propensity in a single model, subsequent studies did not. On analyzing data of a forced desynchrony protocol, the constituent processes could be separated to identify the presence of a nonlinear interaction. ${ }^{17} \mathrm{~A}$ weakness in this model is that it de-emphasizes individual differences in resilience and vulnerability to sleep loss and contradicts recent findings that indicate significant and systematic differences among individuals. This has led to development of more refined biomathematical models in an attempt to predict performance impairment for individuals subjected to total sleep loss ${ }^{22}$. Thus, the understanding of sleep regulation is still under constant refinement with several unanswered questions.

Indian Journal of Sleep Medicine (IJSM), Vol. 9, No. 3, 2014 


\section{Applicability of the Model}

\section{Sleepiness in shift work and jet lag}

Homeostatic sleep drive increases with the duration of prior wakefulness, due to whether acute total sleep deprivation or chronic short sleep schedules. Higher homeostatic sleep drive results in impaired cognition, increased sleepiness, and increased propensity for sleep. Importantly, these circadian and homeostatic processes interact to influence the quality of waking cognition and of sleep. Shift work schedules often require work to occur during the biologic night when the circadian system is promoting sleep, and sleep to occur during the biologic day when the circadian system is promoting wakefulness. The effects of shift work are relatively pronounced, such as a reduction of sleep by $1.5-2 \mathrm{~h}$ when working the night shift schedule and considerable sleepiness (reaching 2-3 min on average for the Multiple Sleep Latency Test and average subjective sleepiness of 7 on the 1-9 level Karolinska Sleepiness Scale). Wakefulness and sleep promoting countermeasures can provide some help to reduce sleepiness and improve sleep, but, currently, effective treatments are lacking that can counteract all of the negative impact that shift work schedules have on human physiology and behavior. Treatment interventions suggested fall into three broad categories: (i) prescribed sleep scheduling, (ii) circadian phase shifting (resetting the clock), and (iii) symptomatic treatment using hypnotic and stimulant medications ${ }^{23}$.

\section{Sleep deprivation}

Studies that have attempted to analyze the relation between the two processes have used a forced desynchrony paradigm, and in the process, discovered effects of sleep deprivation on the processes. In its first applications to sleep ${ }^{24}$, subjects were scheduled to a 28 h sleep-wake cycle. The data showed that maximal sleep propensity coincided with the nadir of the circadian rectal temperature rhythm and rising limb of rectal temperature was paralleled by a gradual decrease in sleep propensity, which reached its lowest level $16 \mathrm{~h}$ after the temperature minimum. This phase corresponds to the habitual bedtime under entrained conditions. When sleep was initiated at this phase, sleep continuity was high. In contrast, poor sleep continuity was observed when sleep was initiated after the temperature minimum. The analysis showed that EEG SWA was determined mainly by homeostatic (i.e., sleep-wake dependent) factors, whereas the REM sleep/non-REM sleep ratio depended on both homeostatic and circadian factors.

\section{Age-related changes in sleepiness}

On the basis of the two-process model, SWA has been used as an objective measure of sleep propensity. In a recent study including normal healthy subjects in threeage groups, it was observed that healthy aging is associated with reduced daytime sleep propensity, sleep continuity, and slow-wave sleep, whereas experimental sleep disruption was found to increase daytime sleep propensity $^{25}$.

\section{Prediction of performance among sleep- deprived individuals}

Recent studies have shown that the two-process model of Borbély could serve as the basis for a better understanding of individualized cognitive daytime performance among sleep-deprived individuals. ${ }^{26,27}$

\section{Conclusion}

The two-process model of sleep, though, first proposed nearly three decades ago, continues to be among the most applicable tools in understanding normal sleep regulation. Current understanding provides amodel in which the interplay between the basic processes " $\mathrm{S}$ " for sleep homeostasis and "C" for circadian propensity for sleepiness and wakefulness, supplemented with newer concepts of "W" process for sleep inertia and " $Z$ " process specifying regional variability across brain areas, determines the nature of the regulation of sleep and wake phases in humans.

\section{References}

1. Borbély AA. A two process model of sleep regulation. Hum Neurobiol 1982;1(3):195-204.

2. Daan S, Beersma DG, Borbély AA. Timing of human sleep: recovery process gated by a circadian pacemaker. Am J Physiol 1984;246(2 Pt 2):R161-R183.

3. Achermann P, Dijk DJ, Brunner DP, Borbély AA. A model of human sleep homeostasis based on EEG slow-wave activity: quantitative comparison of data and simulations. Brain Res Bull 1993;31(1-2):97-113. 
4. Van Dongen HPA, Dinges DF. Investigating the interaction between the homeostatic and circadian processes of sleepwake regulation for the prediction of waking neurobehavioural performance. J Sleep Res $2003 ; 12(3): 181-187$.

5. Borbély AA. Refining sleep homeostasis in the two-process model. J Sleep Res 2009;18(1):1-2.

6. Franken P, Dijk DJ, Tobler I, Borbély AA. Sleep deprivation in rats: effects on EEG power spectra, vigilance states, and cortical temperature. Am J Physiol 1991;261 (1 Pt 2):R198R208.

7. Achermann P, Borbély AA. Mathematical models of sleep regulation. Front Biosci 2003;8:s683-s693.

8. Collop NA, Salas RE, Delayo M, Gamaldo C. Normal sleep and circadian processes. Crit Care Clin 2008;24(3):449460.

9. Carskadon MA, Dement WC. Multiple sleep latency tests during the constant routine. Sleep 1992;15(5):396-399.

10. Durmer JS, Dinges DF. Neurocognitive consequences of sleep deprivation. Semin Neurol 2005;25(1):117-129.

11. Akerstedt T. Sleep/wake disturbances in working life. Electroencephalogr Clin Neurophysiol Suppl 1987;39:360-363

12. Bonnet MH, Arand DL. 24-Hour metabolic rate in insomniacs and matched normal sleepers. Sleep $1995 ; 18(7): 581-588$.

13. Bonnet $\mathbf{M H}$, Arand DL. The consequences of a week of insomnia. Sleep 1996;19(6):453-461.

14. Folkard S, Akerstedt T. Towards a model for the prediction of alertness and/or fatigue on different sleep/wake schedules. In: Oginski A, Polorski J, Rutenfranz J(eds.)Contemporary Advances in Shiftwork Research: Theoretical and Practical Aspects in the Late Eighties. Krakow, Poland: Medical Academy, 1987, pp. 231-240.

15. Zavada A, Strijkstra AM, Boerema AS, Daan S, Beersma DGM. Evidence for differential human slow-wave activity regulation across the brain. J Sleep Res 2009;18(1):3-10.

16. Pittendrigh CS, Daan S. Circadian oscillations in rodents: a systematic increase of their frequency with age. Science $1974 ; 186(4163): 548-550$.
17. Kronauer R. A quantitative model for the effects of light on the amplitude and phase of the deep circadian pacemaker, based on human data. In: Horne J(ed.)Sleep. Bochum,Germany: Pontenagel Press, 1990, pp. 306-309.

18. Beersma DG, Daan S. Generation of activity-rest patterns by dual circadian pacemaker systems: a model. J Sleep Res $1992 ; 1(2): 84-87$.

19. Stephan FK, Zucker I. Circadian rhythms in drinking behavior and locomotor activity of rats are eliminated by hypothalamic lesions. Proc Natl Acad Sci USA $1972 ; 69(6): 1583-1586$.

20. Moore RY. Retinohypothalamic projection in mammals: a comparative study. Brain Res 1973;49(2):403-409.

21. Duffy JF, Czeisler CA. Effect of light on human circadian physiology. Sleep Med Clin 2009;4(2):165-177.

22. Rajaraman S, Gribok AV, Wesensten NJ, Balkin TJ, Reifman J. An improved methodology for individualized performance prediction of sleep-deprived individuals with the two-process model. Sleep 2009;32(10):1377-1392.

23. Sack RL, Auckley D, Auger RR, et al. Circadian rhythm sleep disorders: part I, basic principles, shift work and jet lag disorders. An American Academy of Sleep Medicine review. Sleep 2007;30(11):1460-1483.

24. Dijk DJ, Czeisler CA. Paradoxical timing of the circadian rhythm of sleep propensity serves to consolidate sleep and wakefulness in humans. Neurosci Lett 1994;166(1):6368.

25. Dijk D, Groeger JA, Stanley N, Deacon S. Age-related reduction in daytime sleep propensity and nocturnal slow wave sleep. Sleep 2010;33(2):211-223.

26. Van Dongen HPA, Mott CG, Huang J, et al. Optimization of biomathematical model predictions for cognitive performance impairment in individuals: accounting for unknown traits and uncertain states in homeostatic and circadian processes. Sleep 2007;30(9):1129-1143.

27. Rajaraman S, Gribok AV, Wesensten NJ, Balkin TJ, Reifman J. Individualized performance prediction of sleep-deprived individuals with the two-process model. J Appl Physiol $2008 ; 104(2): 459-468$. 